\title{
DIAGONALIZABLE NORMAL OPERATORS
}

\author{
J. P. WILliams ${ }^{1}$
}

\begin{abstract}
If the image $\varphi(A)$ of a normal operator $A$ on a separable Hilbert space $\mathcal{K}$ is a diagonal operator for some nonzero representation $\varphi$ of $B(\mathcal{K})$ (that annihilates the compact operators), then $A$ must itself be a diagonal operator on $\mathcal{H}$ (with countable spectrum). This yields an "algebraic" characterization of the closure of the range of a derivation induced by a diagonal operator.
\end{abstract}

1. Introduction. If $A$ is a bounded normal operator on a separable Hilbert space $\mathcal{H}$ can one find a faithful $C^{*}$-representation $\varphi$ of the full algebra $\mathscr{B}(\mathcal{H})$ of all bounded linear operators on $\mathcal{H}$ such that the eigenvectors of $\varphi(A)$ span the representation space $\mathcal{H}_{\varphi}$, that is, such that $\varphi(A)$ is a diagonal operator on $\mathcal{H}_{\varphi}$ ? The question was raised by B. E. Johnson in connection with some work on derivations [4]. Our purpose here is to supply the answer: only if $A$ is already a diagonal operator on $\mathcal{K}$. We show also that $\varphi(A)$ is a diagonal operator for a nonzero representation $\varphi$ of $\mathscr{B}(\mathcal{H C})$ that annihilates the compact operators if and only if $A$ is a diagonal operator with countable spectrum.

The nondiagonalizability result as just stated hardly seems surprising. But it is interesting to note that the proof seems to require a deep result only recently discovered. Moreover, Johnson's question is reasonable because an affirmative answer would provide a satisfying result about derivations, or better, because Berberian [1] has shown that one can find a faithful representation $\varphi$ of $\mathscr{B}(\mathcal{H})$ such that each point of the spectrum of $\varphi(A)$ is an eigenvalue.

2. Diagonalizability. For $A \in \mathscr{B}(\mathcal{H})$ we shall denote by $\delta_{A}$ the inner derivation $X \rightarrow A X-X A$ on $\mathscr{B}(\mathcal{H})$ and by $\mathscr{K}$ the ideal of compact operators on $\mathcal{H}$.

THEOREM 1. Let $A$ be a normal operator on a separable Hilbert space $\mathcal{H}$. The following conditions are equivalent:

(1) There exists a nonzero representation $\varphi$ of $\mathscr{B}(\mathcal{H})$ on a Hilbert space $\mathcal{H}_{\varphi}$ (not necessarily faithful) such that $\varphi(A)$ is a diagonal operator on $\mathcal{H}_{\varphi}$.

(2) $A$ is a diagonal operator on $\mathcal{H}$.

(3) Each positive operator in the norm closure $\mathcal{R}_{(}\left(\delta_{A}\right)^{-}$of the range of $\delta_{A}$ is compact.

(4) Each projection in $\Re\left(\delta_{A}\right)^{-}$has finite rank.

Proof. The implications $(2) \Rightarrow(1)$ and $(3) \Rightarrow(4)$ are trivial. Suppose (1)

Received by the editors November 13, 1974.

AMS (MOS) subject classifications (1970). Primary 46L05, 47B15, 47A50, $47 \mathrm{~B} 47$.

Key words and phrases. Representations of $C^{*}$-algebras, normal operators, range of a derivation.

${ }^{1}$ Research supported by NSF GP 28601. 
holds so that $\varphi(A) e_{i}=\lambda_{i} e_{i}$ for some orthonormal basis $\left\{e_{i}\right\}$ of $\mathcal{H}_{\varphi}$, and let $Z=\lim \delta_{A}\left(X_{n}\right)$ be a positive operator in $\mathscr{R}\left(\delta_{A}\right)^{-}$. Then

$$
\begin{aligned}
\left(\varphi(Z) e_{i}, e_{i}\right) & =\lim \left[\left(\varphi(A) \varphi\left(X_{n}\right) e_{i}, e_{i}\right)-\left(\varphi\left(X_{n}\right) \varphi(A) e_{i}, e_{i}\right)\right] \\
& =\lim \left[\lambda_{i}\left(\varphi\left(X_{n}\right) e_{i}, e_{i}\right)-\lambda_{i}\left(\varphi\left(X_{n}\right) e_{i}, e_{i}\right)\right]=0
\end{aligned}
$$

Hence $\sqrt{\varphi(Z)} e_{i}=0$ for each $i$ so that $\varphi(Z)=0$. Thus $Z \in \operatorname{ker}(\varphi) \subset \mathscr{K}$ because $\mathcal{H}$ is separable. Thus (1) $\Rightarrow(3)$.

We complete the proof by showing $(4) \Rightarrow(2)$, or what is the same, that if $A$ is not a diagonal operator then $\Re\left(\delta_{A}\right)^{-}$contains an infinite rank projection. By replacing $A$ with its restriction to the orthocomplement of the span of its eigenvectors, there is no loss of generality in assuming that $A$ itself has no eigenvectors.

Let $B$ be the direct sum of countably many copies of $A$ acting in the usual way on the space $\tilde{\mathcal{H}}=\mathscr{H} \oplus \mathscr{H} \oplus \mathcal{H} \oplus \cdots$. Then by a theorem of I. D. Berg [2] there is a unitary transformation $U$ mapping $\mathcal{H}$ onto $\tilde{H}$ and a compact operator $K$ on $\mathcal{H}$ such that $U^{-1} B U=A+K$.

Now $A$ and $B$ have no eigenvalues and consequently $\Re\left(\delta_{A}\right)^{-}$and $\Re\left(\delta_{B}\right)^{-}$ respectively contain all the compact operators on $\mathcal{H}$ and $\tilde{\mathcal{H}}$ respectively [6]. Hence $\mathcal{R}\left(\delta_{A}\right)^{-}=U^{-1} \mathfrak{R}\left(\delta_{B}\right)^{-} U$. Also, for the same reason, if $P_{0}$ is any nonzero projection of finite rank, we can choose a sequence $X_{n} \in \mathscr{B}(\mathcal{H})$ such that $\left\|\delta_{A}\left(X_{n}\right)-P_{0}\right\| \leqslant n^{-1}$. Let $\tilde{X}_{n}$ be the direct sum of countably many copies of $X_{n}$ and let $\tilde{P}$ be the direct sum of as many copies of $P_{0}$. Then $\left\|\delta_{B}\left(\tilde{X}_{n}\right)-\tilde{P}\right\|$ $\leqslant n^{-1}$ so that $\tilde{P} \in \mathcal{R}\left(\delta_{B}\right)^{-}$, and consequently, $P=U^{-1} \tilde{P} U$ is a projection of infinite rank in $\mathcal{R}\left(\delta_{A}\right)^{-}$.

3. Essential diagonalizability. A diagonal operator $A$ on a separable space $\mathcal{H}$ has only countably many eigenvalues of course, but the spectrum itself can be any prescribed compact subset of the plane. However, if $A$ is also diagonalizable by a representation of the Calkin algebra, there is a severe restriction on the spectrum. The first assertion of the next theorem was pointed out to me by $\mathrm{C}$. Foiaş.

THEOREM 2. Let $A$ be a normal operator on a separable Hilbert space $\mathcal{H}$.

(1) If $\varphi(A)$ is a diagonal operator for some nonzero representation $\varphi$ of $\mathscr{B}(\mathcal{H})$ with $\varphi(\mathscr{K})=0$, then the spectrum of $A$ is countable.

(2) Conversely, if $A$ has countable spectrum, then $\varphi(A)$ is a diagonal operator for any nonzero representation $\varphi$ of $\mathscr{B}(\mathcal{H})$.

Proof. (1) Suppose that the spectrum $\sigma(A)$ of $A$ is not countable. Then there is a continuous measure $\mu$ with support contained in $\sigma(A)[5$, p. 176]. (For example, take $\mu=\nu \circ f^{-1}$ where $\nu$ is Haar measure on the compact abelian group $G=\{0,1\}^{\mathbf{N}}$ and $f$ is a homeomorphism from $G$ into $\sigma(A)$.) Let $B_{0}$ be the operator defined by multiplication by the independent variable in $L^{2}(\mu)$. Then $A$ and $B=B_{0} \oplus A$ have the same essential spectrum, so that by Berg's theorem [2] there is a unitary operator $U$ from $L^{2}(\mu) \oplus \mathcal{H}$ onto $\mathcal{H}$ and a compact operator $K$ with $U B U^{-1}=A+K$. But then $\varphi\left(U B U^{-1}\right)=$ $\varphi(A+K)=\varphi(A)$ is diagonal so that (Theorem 1) $U B U^{-1}$, and therefore $B$ itself, is diagonal. This is a contradiction since $B_{0}$ has no eigenvalues. 
(2) Suppose that $A$ is a diagonal operator on $\mathcal{H}$ with countable spectrum and let $\varphi$ be a nonzero representation of $\mathscr{B}(\mathcal{H})$ on a Hilbert space $\mathcal{H}_{\varphi}$. Let $\mathfrak{N}=\varphi(1) \mathcal{H}_{\varphi}$. If $X \in \mathscr{B}(\mathcal{H})$ then $\varphi(X)=\varphi_{0}(X) \oplus 0$ on $\mathcal{H}_{\varphi}=\mathfrak{K} \oplus \mathfrak{N}^{\perp}$ so that $\varphi_{0}$ is a representation of $\mathscr{B}(\mathcal{H})$ on $\mathscr{N}$ and $\varphi_{0}(1)$ is the identity operator on T. In particular, the operator $\varphi_{0}(A)$ has spectrum contained in $\sigma(A)$ and is therefore countable. It suffices to show, therefore, that a normal operator $B$ on a Hilbert space $\pi$ having countable spectrum is diagonal. This fact is well known: if $\Re_{0}$ is the span of the eigenvectors of $B$ then $\Re_{1}=\Re \ominus \Re_{0}$ reduces to 0 ; otherwise $\sigma\left(B \mid \Re_{1}\right)$, being countable, must have an isolated point and this is necessarily an eigenvalue of $B$.

REMARK 1. L. G. Brown has observed that Theorem 2 is valid as stated with the weaker hypothesis that the operator $A$ is essentially normal, i.e., that $\pi(A)=A+\mathscr{K}$ is a normal element of the quotient $\mathscr{B}(\mathscr{K}) / \mathscr{K}:$ if $\sigma(A)$ is uncountable choose a normal operator $B_{0}$ with no eigenvalues such that $\sigma\left(B_{0}\right) \subset \sigma(A)$ and let $B=A \oplus B_{0} \oplus B_{0} \oplus \cdots$. Then $\mathcal{R}\left(\delta_{B}\right)^{-}$contains a projection of infinite rank so that $\varphi(B)$ is not a diagonal operator for any nonzero representation $\varphi$ of $\mathscr{B}(\mathcal{H})$. Hence if $\varphi(\mathscr{K})=0$ then $\varphi(A)$ is also not a diagonal operator because $\pi(A)$ and $\pi(B)$ are unitarily equivalent [3].

2. After this paper was completed the author discovered a preprint of John G. Aiken, An application of direct integral theory to a question of Calkin, [Notices Amer. Math. Soc. 21 (1974), A493]. Aiken constructs a diagonal operator $A$ such that $T_{u}(A)$ is not a diagonal operator for any of the "generalized limit" representations $T_{u}$ of $\mathscr{B}(\mathcal{H}) / \mathscr{K}$ introduced by J. W. Calkin, thereby answering a question explicitly(!) raised in the latter's famous paper [Ann. of Math. (2) 42 (1941), 839-873. MR 3, 208].

\section{REFERENCES}

1. S. K. Berberian, Approximate proper vectors, Proc. Amer. Math. Soc. 13 (1962), 111-114. MR 24 \# A3516.

2. I. D. Berg, An extension of the Weyl-von Neumann theorem to normal operators, Trans. Amer. Math. Soc. 160 (1971), 365-371. MR 44\#840.

3. L. G. Brown, R. G. Douglas and P. A. Fillmore, Unitary equivalence modulo the compact operators and extensions of $C^{*}$-algebras, Proc. Conf. on Operator Theory, Lecture Notes in Math., vol. 345, Springer-Verlag, New York, 1973.

4. B. E. Johnson and J. P. Williams, The range of a normal derivation, Pacific J. Math. 56 (1975).

5. W. Rudin, Real and complex analysis, McGraw-Hill, New York, 1966. MR 35\# 1420.

6. J. P. Williams, On the range of a derivation, Pacific J. Math. 38 (1971), 273-279. MR $46 \# 7923$.

Department of Mathematics, Indiana University, Bloomington, Indiana 47401 